\title{
Multiple Logic Gates Based on Electrically Wired Surface-Reconstituted Enzymes
}

\author{
Marcos Pita and Evgeny Katz \\ Department of Chemistry and Biomolecular Science, Clarkson University, Potsdam NY 13699-5810, \\ USA
}

Electrode modification: A gold electrode ( $\mathrm{Au}$ wire $0.5 \mathrm{~mm}$ diameter, active area ca. 0.2 $\mathrm{cm}^{2}$ ) was used for the measurements. The electrode was cleaned according to the previously reported procedure ${ }^{1}$ by boiling in $2 \mathrm{M} \mathrm{KOH}$ for $1 \mathrm{~h}$, followed by rinsing with distilled water and then stored in concentrated sulfuric acid. Prior to the modification, the electrode was rinsed with water, soaked for $10 \mathrm{~min}$ in concentrated nitric acid, and rinsed again with water. The Au electrode was modified with amino groups by soaking in a solution of $0.05 \mathrm{M}$ cystamine in water for $1 \mathrm{~h}$ followed by rinsing with water to remove the physically adsorbed cystamine. ${ }^{1}$ The cystamine-modified Au electrode was functionalized with a pyrroloquinoline quinone (PQQ, 1) monolayer $^{2}$ by reacting with 3 $\mathrm{mM}$ solution of PQQ (Sigma-Aldrich) in 0.1 M HEPES-buffer ( $N$-2hydroxyethylpiperazine- $N$ '-2-ethanesulfonic acid), $\mathrm{pH} 7.2$, in the presence of $5 \mathrm{mM} 1$ ethyl-3-(3-(dimethylamino)propyl)carbodiimide (EDC Sigma-Aldrich) for 2 hours. The PQQ-modified electrode was further reacted with $1 \mathrm{mM} 3$-aminophenylboronic acid (2, Sigma-Aldrich) solution in 0.1 M HEPES-buffer, $\mathrm{pH} 7.2$, in the presence of $5 \mathrm{mM}$ EDC for 2 hours, followed by rinsing with distilled water. The surface-confined phenylboronic groups were used as specific linkers to bind covalently FAD (3) cofactor through vicinal hydroxyl groups. The PQQ-FAD-modified Au-electrode was reacted with $1 \mathrm{mg} \mathrm{mL}$ apo-GOx (prepared by previously reported method $^{3}$ ) in $0.1 \mathrm{M}$ phosphate buffer, $\mathrm{pH}$ 7.0, for 4 hours at room temperature to yield a GOx-reconstituted electrode. ${ }^{4}$ The GOxmodified electrode was further reacted with $1 \mathrm{mM}$ solution of microperoxidase-11 (MP11, 4, Sigma-Aldrich) in 0.1 M HEPES-buffer, $\mathrm{pH} 7.2$, in the presence of $5 \mathrm{mM}$ EDC for 2 hours, followed by rinsing with $0.1 \mathrm{M}$ phosphate buffer, $\mathrm{pH}$ 7.0. The procedure was similar to one published elsewhere ${ }^{5}$ for the immobilization of MP-11 on an aminofunctionalized electrode, and it resulted in the co-immobilization of MP-11 on the GOx- 
modified electrode. The stepwise modification procedure and the structures of all chemicals are shown in SI-Scheme 1.

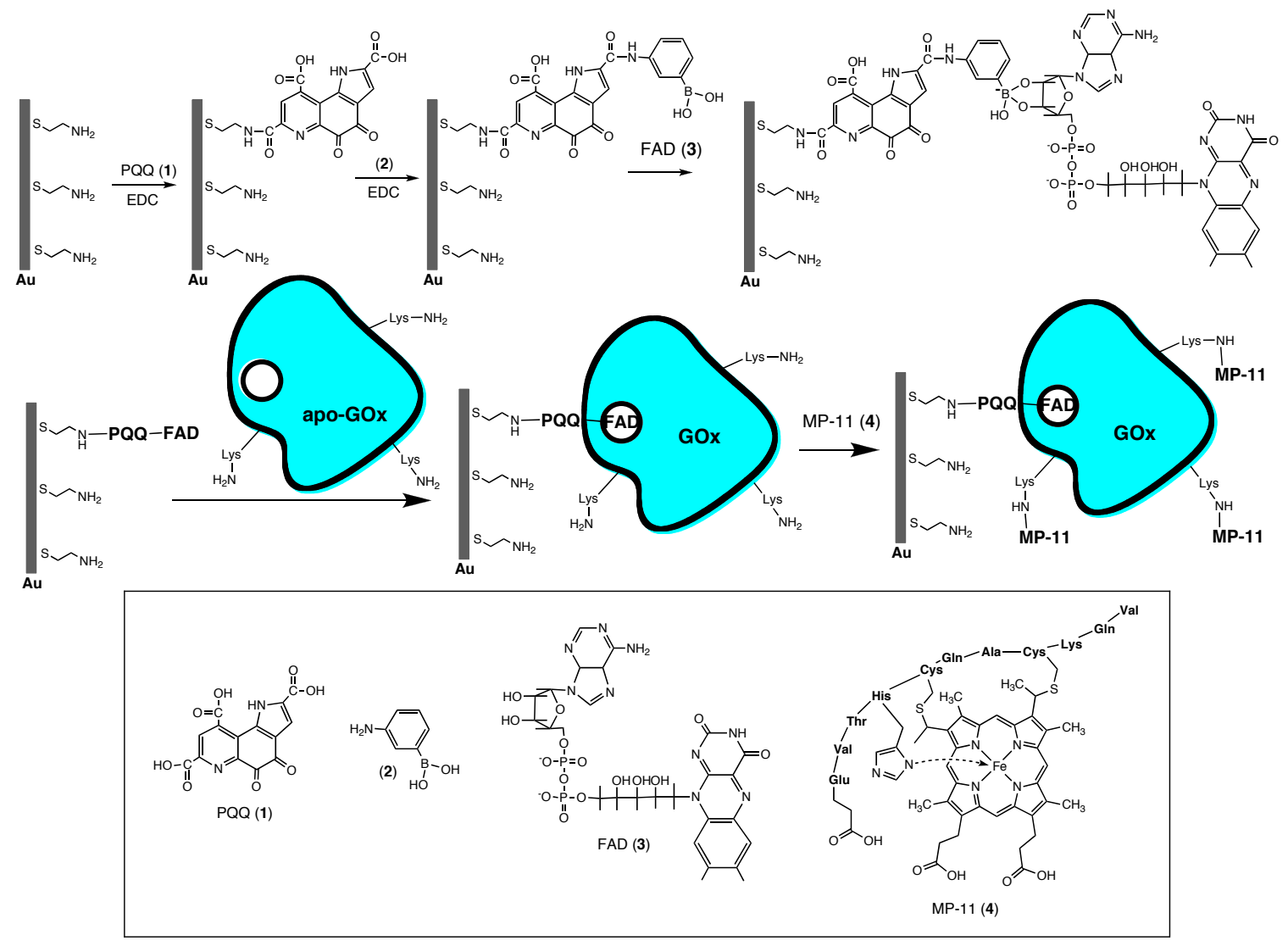

SI-Scheme 1: Stepwise modification of the electrode surface to yield the electrode biocatalytically active for the glucose oxidation and for $\mathrm{H}_{2} \mathrm{O}_{2}$ reduction/oxidation. 
Electrochemical measurements: Electrochemical measurements were performed using an electrochemical analyzer (Autolab PGSTAT12, Eco Chemie B.V.). The measurements were carried out at an ambient temperature $\left(23 \pm 2^{\circ} \mathrm{C}\right)$ in a conventional electrochemical cell consisting of the GOx/MP-11-modified Au electrode as a working electrode (active area ca. $02 \mathrm{~cm}^{2}$ ), a Pt-grid auxiliary electrode, and a saturated $\mathrm{Ag} / \mathrm{AgCl}$ reference electrode. All potentials are reported with respect to this reference electrode. The cell was placed in a grounded Faraday cage. The cyclic voltammograms, differential pulse voltammograms and current values at fixed potentials were measured in $0.1 \mathrm{M}$ phosphate buffer, $\mathrm{pH}$ 7.0, in the absence or presence of $5 \mathrm{mM}$ glucose or/and $5 \mathrm{mM} \mathrm{H}_{2} \mathrm{O}_{2}$.

\section{Electrochemical characterization of the electrode upon its stepwise modification:}

Differential pulse voltammograms (DPVs) were recorded after all modification steps to follow the chemical changes at the surface (Figure SI-1). The peak $\left(E_{p}=-0.1\right.$ V) corresponding to the redox process of the surface-confined PQQ appeared after the covalent attachment of PQQ to the cystamine monolayer (Fig. SI-1, curve a) and it is observed almost unchanged in all DPVs reflecting external positioning of the redox groups vs. the attached protein molecules. Binding of the FAD cofactor units resulted in the appearance of the peak $\left(\mathrm{E}_{\mathrm{p}}=-0.38 \mathrm{~V}\right)$ corresponding to the FAD redox process (Fig. SI-1, curve b). Similar peak current values for the PQQ and FAD redox processes reflect their 1:1 ratio at the modified surface. Reconstitution of apo-GOx on the FAD-PQQmodified electrode resulted in the depletion of the FAD peak in the DPV (Fig. SI-1, curve c) similarly to the results observed before. ${ }^{4}$ Further modification of the electrode surface with MP-11 resulted in the appearance of the peak corresponding to the redox process of the heme associated with MP-11 ( $E_{p}=-0.35$ V) (Fig. SI-1, curve d). Cyclic voltammograms for the electrodes after the GOx reconstitution and after the MP-11 coimmobilization were also obtained (Fig. SI-2, curves a and b, respectively) and used to derive the surface concentration of MP-11 on the electrode surface. 


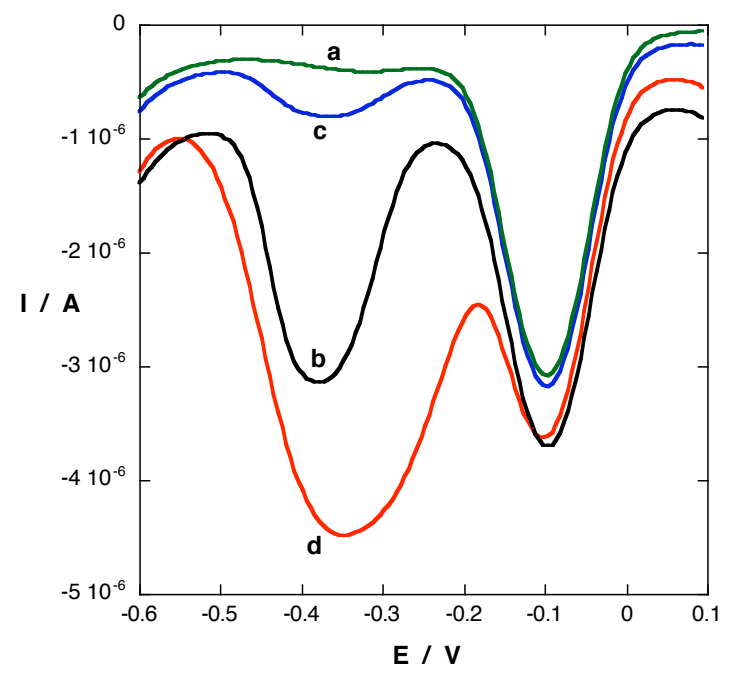

Figure SI-1: Differential pulse voltammograms obtained upon stepwise modification of the $\mathrm{Au}$ electrode: a) PQQ-functionalized electrode, b) PQQ-FAD-functionalized electrode, c) PQQ-FAD-functionalized electrode after the reconstitution of GOx, d) PQQFAD-functionalized electrode with the reconstituted GOx and covalently bound MP-11. The measurements were performed in $0.1 \mathrm{M}$ phosphate buffer, $\mathrm{pH} 7.0$, with the potential scan rate $10 \mathrm{mV}^{-1}$.

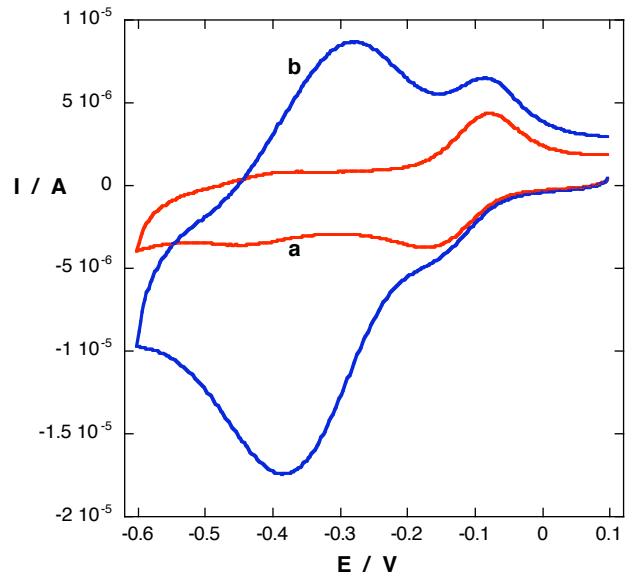

Figure SI-2: Cyclic voltammograms of: a) the GOx-reconstituted electrode, b) the GOxreconstituted electrode with the covalently bound MP-11. The measurements were performed in $0.1 \mathrm{M}$ phosphate buffer, $\mathrm{pH} 7.0$, with the potential scan rate $200 \mathrm{mV} \mathrm{s}^{-1}$. 


\section{References:}

1. Katz, E.; Solov'ev, A.A. J. Electroanal. Chem. 1990, 291, 171-186.

2. Katz, E.; Schlereth, D.D.; Schmidt, H.-L. J. Electroanal. Chem. 1994, 367, 59-70.

3. Katz, E.; Riklin, A.; Heleg-Shabtai, V.; Willner, I.; Bückmann, A.F. Anal. Chim. Acta 1999, 385, 45-58.

4. Zayats, M.; Katz, E.; Willner, I. J. Am. Chem. Soc. 2002, 124, 14724-14735.

5. Lötzbeyer, T.; Schuhmann, W.; Katz, E.; Falter, J.; Schmidt, H.-L. J. Electroanal. Chem. 1994, 377, 291-294. 